\title{
Ballistic Trajectory Modeling for Missile with Deflectable Nose
}

\author{
Yongjie XU*, Zhijun WANG*, Fangdong DONG** \\ *College of Mechatronic Engineering, North University of China, 030051 Taiyuan, P.R. China, \\ E-mail: yongqiang515@nuc.edu.cn (Corresponding Author) \\ *College of Mechatronic Engineering, North University of China, 030051 Taiyuan, P.R. China, E-mail: wzj@nuc.edu.cn \\ **Science and Technology on Transient Impact Laboratory, 102202 Beijing, P.R. China, E-mail: dfd0415@126.com

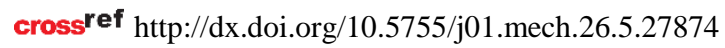

\section{Introduction}

With a continuous development of weapons and military equipment, tectonic changes are observed in the state-of-the-art war strategies, which necessitate precision strikes on the enemy military objects with the minimization of collateral damage to civilians and civilian infrastructure. Conventional projectiles like shells and mines are prone to deviation from the intended target and have limited hit probability due to such disturbance factors as fabrication/mismatch errors, initial direction and/or velocity errors, and stochastic wind effect. To improve the shooting dispersion and reduce the effect of the above interference factors, different methods of improving the structure of guns or ammunition are applied but their effectiveness falls behind the operational requirements of modern warfare [1-2]. Given this, an innovative type of ammunition, which would be relatively cheap and combine high precision with enhanced damage efficiency, is very topical. Such an option is expected to be provided by the trajectory correction technology, which ensures the capability of firing range correction of a projectile reduces the initial shooting dispersion and improves the hit probability through several ballistic corrections.

Aircraft with a deflectable nose part is a lucrative fast-response ballistic control method, which has been introduced for steering an aircraft while coasting at very high speed, which approach is also applicable for steering a projectile in powered flight [3]. Characteristics of the flight flow field and pressure distribution of a projectile with deflectable nose control has been studied through methods of wind tunnel test [4-7] and computational fluid dynamic simulation [8-11]. The design method of the deflectable nose control system] has been studied by numerical simulations [12-14].

Research on the creative intelligent control technology has high scientific merits and practical value, whereas the external ballistics plays a crucial role in this modern missile control technology. Research on the ballistic control technology has significant practical importance and broad application prospect sin the aspect of improving the ammunition equipment level.

\section{Dynamic model for projectile with deflectable nose}

\subsection{Coordinate systems}

Although the governing equations of projectile motion are invariant of the applied coordinate systems, a proper selection of the latter can minimize the simulation problems and their solutions. References [15-17] adopt the conventional coordinate systems, which are used for the dynamic modeling. These coordinate systems are schematically presented in Fig.1.

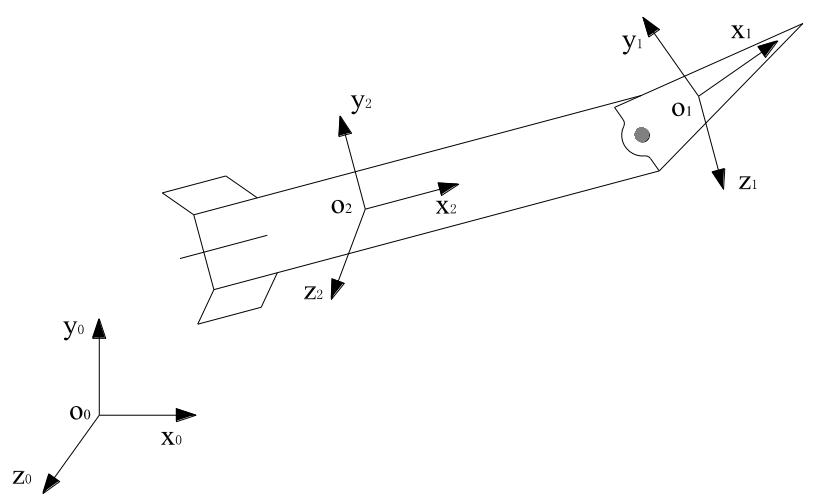

Fig. 1 Schematic diagram of coordinate systems

1 . The ground coordinate system $O_{0} x_{0} y_{0} z_{0}$ is an inertial reference coordinate, which is fixed on the earth surface and is mainly used to determine the mass center position and orientation of the projectile in space.

2. Projectile-nose coordinate system $O_{1} x_{1} y_{1} z_{1}$ is fixed to the projectile nose, which is a moving coordinate system.

3. Projectile-body coordinate system $\mathrm{O}_{2} x_{2} y_{2} z_{2}$ is attached to the projectile body, which is a moving coordinate system.

4. Velocity coordinate system $O_{3} x_{3} y_{3} z_{3}$ is fixed to the velocity vector, which is a moving coordinate system depicted in Fig. 2.

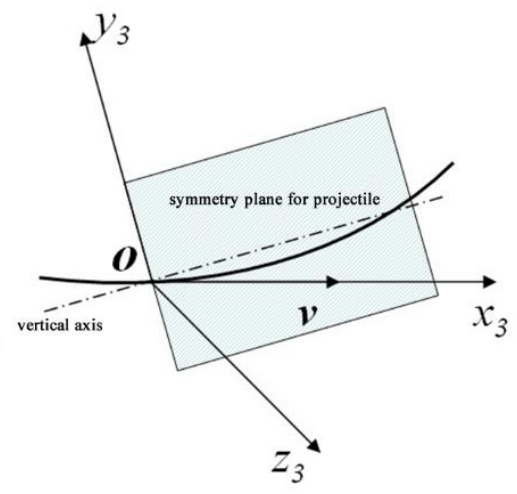

Fig. 2 Schematic diagram of velocity coordinate system

The following angles are involved: $\alpha$ is the projectile nose high-low deflection angle, $\beta$ is the projectile nose 
directional deflection angle *(both are defined in the projectile nose coordinate system); $\theta$ is the pitch angle, $\varphi$ is the yaw angle, $\delta_{1}$ is the high-low attack angle , and $\delta_{2}$ is the directional attack angle (these angles are defined in the projectile body coordinate system); $\psi_{1}$ is the trajectory inclination angle, $\psi_{2}$ is the trajectory deflection angle ( these angles are set in the velocity coordinate system).

2.2. Conversion relationship between the coordinate systems

At any instant of the projectile motion, each coordinate system mentioned above has its specific orientation in space, and there are specific formulas for the conversion of coordinates from one system to others. When establishing the scalar equations of the projectile motion, the parameters defined by different coordinates must be projected onto the same coordinates [18-19]. Therefore, the coordinate transformation matrix is essential in the conversion of parameters specified in their generated coordinate into a new coordinate.

Transformation matrixes of different coordinate systems are respectively as follows.

$$
\begin{aligned}
A_{02} & =\left[\begin{array}{lll}
\cos \varphi \cdot \cos \theta & \cos \varphi \cdot \sin \theta & \sin \varphi \\
-\sin \theta & \cos \theta & 0 \\
-\sin \varphi \cdot \cos \theta & -\sin \varphi \cdot \sin \theta & \chi \operatorname{oo\sigma } \varphi
\end{array}\right], \\
A_{03} & =\left[\begin{array}{lll}
\cos \psi_{2} \cdot \cos \psi_{1} & \cos \psi_{2} \cdot \sin \psi_{1} & \sin \psi_{2} \\
-\sin \psi_{1} & \cos \psi_{1} & 0 \\
-\sin \psi_{2} \cdot \cos \psi_{1} & -\sin \psi_{2} \cdot \sin \psi_{1} & \cos \psi_{2}
\end{array}\right], \\
A_{23} & =\left[\begin{array}{lll}
\cos \delta_{2} \cdot \cos \delta_{1} & \cos \delta_{2} \cdot \sin \delta_{1} & \sin \delta_{2} \\
-\sin \delta_{1} & \cos \delta_{1} & 0 \\
-\sin \delta_{2} \cdot \cos \delta_{1} & -\sin \delta_{2} \cdot \sin \delta_{1} & \cos \delta_{2}
\end{array}\right] .
\end{aligned}
$$

\subsection{Force and moment analysis}

A projectile is composed of a warhead and a projectile body, regardless of the spinning, whereas forces and moments acting on it are considered to be applied to the centroid of each part of the projectile. Besides, subscripts $i=1$ and $i=2$ correspond to the projectile body and projectile nose, respectively.

$G$ is a gravitational force in the ground coordinate system, which is expressed as:

$$
\left[m_{i} \cdot g\right]=\left[\begin{array}{l}
0 \\
-m_{i} \cdot g \\
0
\end{array}\right]
$$

where: $m$ is mass and $g$ is gravity acceleration.

$R_{x}$ is the aerodynamic drag, which is directed along the negative $\mathrm{O}_{3} \mathrm{X}_{3}$ axis in the velocity coordinate system.

$$
\left[\boldsymbol{R}_{x i}\right]=A_{03} \cdot\left[\begin{array}{l}
-R_{x i} \\
0 \\
0
\end{array}\right]=-R_{x i} \cdot\left[\begin{array}{l}
\cos \psi_{1} \cdot \cos \psi_{2} \\
-\sin \psi_{1} \\
-\sin \psi_{2} \cdot \cos \psi_{1}
\end{array}\right]
$$

$R_{y}$ is the aerodynamic lift, which direction coincides with the positive $\mathrm{O}_{3} Y_{3}$ axis in the velocity coordinate system.

$$
\left[\boldsymbol{R}_{y i}\right]=A_{03} \cdot\left[\begin{array}{l}
0 \\
R_{y i} \\
0
\end{array}\right]=R_{y i} \cdot\left[\begin{array}{l}
\cos \psi_{2} \cdot \sin \psi_{1} \\
\cos \psi_{1} \\
-\sin \psi_{1} \cdot \sin \psi_{2}
\end{array}\right]
$$

$F_{p}$ is the engine thrust, which direction coincides with the positive $\mathrm{O}_{2} \mathrm{X}_{2}$ axis in the projectile-body coordinate system.

$$
\boldsymbol{F}_{p}=A_{02} \cdot\left[\begin{array}{l}
F_{p} \\
0 \\
0
\end{array}\right]=F_{p} \cdot\left[\begin{array}{l}
\cos \theta \cdot \cos \phi \\
-\sin \phi \\
-\cos \theta \cdot \sin \phi
\end{array}\right] .
$$

$M_{z}$ is a static moment, which is expressed as:

$$
\mathbf{M}_{z}=\frac{\rho \cdot \mathbf{V}^{2}}{2} \cdot S \cdot l \cdot m_{Z}
$$

where: $\rho$ is air density; $V$ is the speed of projectile motion; $S$ is a reference area and which is equal to the projectile cross sectional; $l$ is a reference length which is equal to the projectile diameter, and $m_{z}$ is static moment coefficient.

The static moment direction coincides with the $Z$ axis of the projectile-nose or projectile-body coordinate systems and is derived as follows:

$$
\left[\mathbf{M}_{z i}\right]=\left[\begin{array}{l}
0 \\
0 \\
M_{z i}
\end{array}\right]
$$
pressed as:

$M_{z z}$ is equatorial damping moment, which is ex-

$$
\mathbf{M}_{Z Z}=\frac{\rho \cdot \mathbf{V}^{2}}{2} \cdot S \cdot l \cdot m_{Z Z},
$$

where: $m_{z z}$ is equatorial damping moment coefficient.

The equatorial damping moment direction coincides with the $Y$-axis of the projectile-nose or projectilebody coordinate systems and is described as:

$$
\left[\mathbf{M}_{y i}\right]=\left[\begin{array}{l}
0 \\
-M_{y i} \\
0
\end{array}\right]
$$

\section{Equations for the two-rigid-body trajectory model}

\subsection{Basic assumption}

The equations of the trajectory model are set under the following underlying assumptions.

The projectile is axisymmetric and consists of a warhead and a projectile body. Its dimensions and design 
imply a uniform mass distribution. The nose can deflect relative to the projectile body in yaw and pitch directions. The rotation of the projectile is not considered and is regardless of the deflection process, and the mass of the projectile body is evenly distributed in the transverse or longitudinal symmetry plane after the nose deflected. Meteorological conditions shall be standard without wind or rain disturbance factors.

\subsection{Centroid movement equation}

The motion of projectile's mass center relative to the inertial coordinate system obeys Newton's law as follows:

$$
m \cdot \frac{d \mathbf{V}}{d t}=\sum \mathbf{F}
$$

For the projectile nose and projectile body, Eqs. (13-14) can be derived:

$$
\begin{aligned}
& m_{1} \cdot \frac{d \mathbf{V}}{d t}=\sum \mathbf{F}_{1}=\mathbf{F}_{12}+\mathbf{R}_{x 1}+\mathbf{R}_{y 1}, \\
& m_{2} \cdot \frac{d \mathbf{V}}{d t}=\sum \mathbf{F}_{2}=\mathbf{F}_{21}+\mathbf{R}_{x 2}+\mathbf{R}_{y 2},
\end{aligned}
$$

where: $\mathbf{F}_{12}$ is the acting force of the projectile-body on the projectile-nose; $\mathbf{F}_{21}$ is the acting force of the projectile-nose on the projectile-body. They have identical absolute values but opposite directions.

Combining the force analysis results above, the dynamic equations of the center of mass motion (15) can be obtained:

$$
\begin{aligned}
& \frac{d V_{x}}{d t}=\frac{F_{p}}{m} \cdot \cos \theta \cdot \cos \varphi-\frac{R_{x}}{m} \cdot \cos \psi_{1} \cdot \cos \psi_{2}+\frac{R y}{m} \cdot \sin \psi_{1} \cdot \cos \psi_{2} \\
& \frac{d V_{y}}{d t}=\frac{-F_{p}}{m} \cdot \sin \theta+\frac{R_{x}}{m} \cdot \sin \psi_{1}+\frac{R y}{m} \cdot \cos \psi_{1}-g \\
& \frac{d V_{z}}{d t}=\frac{-F_{p}}{m} \cdot \cos \theta \cdot \sin \varphi+\frac{R_{x}}{m} \cdot \cos \psi_{1} \cdot \sin \psi_{2}-\frac{R y}{m} \cdot \sin \psi_{1} \cdot \sin \psi_{2}
\end{aligned}
$$

where: $m$ is the projectile mass $\left(m=m_{1}+m_{2}\right) ; \mathbf{R}_{\mathrm{x}}$ is the total aerodynamic drag of the projectile $\left(\mathbf{R}_{\mathrm{x}}=\mathbf{R}_{\mathrm{x} 1}+\mathbf{R}_{\mathrm{x} 2}\right.$; similarly, $\left.\mathbf{R}_{\mathrm{y}}=\mathbf{R}_{\mathrm{y} 1}+\mathbf{R}_{\mathrm{y} 2}\right)$.

The centroid motion kinematic Eq. (16) can be derived from the velocity vector components along the three axes of the ground coordinate system.

$$
\left.\begin{array}{l}
\frac{d x}{d t}=V_{x}=V \cdot \cos \psi_{1} \cdot \cos \psi_{2} \\
\frac{d y}{d t}=V_{y}=V \cdot \sin \psi_{1} \cdot \cos \psi_{2} \\
\frac{d z}{d t}=V_{z}=V \cdot \sin \psi_{2}
\end{array}\right\} .
$$

\subsection{Motion equation of rotation about the centroid}

The rotation of a projectile around its center of mass obeys the Theorem of the Moment of Momentum, which is given as follow.

$$
\frac{d \mathbf{G}}{d t}+\boldsymbol{\omega} \times \mathbf{G}=\sum \mathbf{M}
$$

where: $\mathbf{G}$ is the momentum moment vector; $\boldsymbol{\omega}$ is the angular velocity vector and $\sum \mathbf{M}$ is the resultant moment.

For the projectile nose, Eqs. (18-20) can be derived:

$$
\begin{aligned}
& {\left[\mathbf{G}_{1}\right]=\left[\mathbf{J}_{1}\right] \cdot\left[\boldsymbol{\omega}_{1}\right]=} \\
& =\left[\begin{array}{lll}
C_{1} & 0 & 0 \\
0 & A_{1} & 0 \\
0 & 0 & A_{1}
\end{array}\right] \cdot\left[\begin{array}{l}
\dot{\alpha} \cdot \sin \beta \\
-\dot{\beta} \\
\dot{\alpha} \cdot \cos \beta
\end{array}\right]=\left[\begin{array}{l}
C_{1} \cdot \dot{\alpha} \cdot \sin \beta \\
-A_{1} \cdot \dot{\beta} \\
A_{1} \cdot \alpha \cdot \cos \beta
\end{array}\right],
\end{aligned}
$$

$$
\begin{aligned}
& {\left[\frac{d \mathbf{G}_{1}}{d t}\right]=\left[\begin{array}{c}
C_{1} \cdot(\ddot{\alpha} \cdot \sin \beta+\dot{\alpha} \cdot \dot{\beta} \cdot \cos \beta) \\
-A_{1} \cdot \ddot{\beta} \\
A_{1} \cdot(\ddot{\alpha} \cdot \cos \beta-\dot{\alpha} \cdot \dot{\beta} \cdot \sin \beta)
\end{array}\right],} \\
& {\left[\boldsymbol{\omega} \times \mathbf{G}_{1}\right]=\left[\begin{array}{l}
\omega_{1 y} \cdot G_{z 1}-\omega_{1 z} \cdot G_{y 1} \\
\omega_{1 z} \cdot G_{x 1}-\omega_{1 x} \cdot G_{z 1} \\
\omega_{1 x} \cdot G_{y 1}-\omega_{1 y} \cdot G_{x 1}
\end{array}\right]=} \\
& 0 \\
& =\left[\begin{array}{l}
\left(C_{1}-A_{1}\right) \cdot \dot{\alpha}^{2} \cdot \sin \beta \cdot \cos \beta \\
\left(C_{1}-A_{1}\right) \cdot \dot{\alpha} \cdot \dot{\beta} \cdot \sin \beta
\end{array}\right],
\end{aligned}
$$

where: $J_{1}$ is the matrix of the moment of inertia; $C_{1}$ is the equatorial moment of inertia and $A_{1}$ is the polar moment of inertia for the projectile nose.

For the projectile body, Eqs. (21-23) can be derived:

$$
\begin{aligned}
& {\left[\mathbf{G}_{2}\right]=\left[\mathbf{J}_{2}\right] \cdot\left[\boldsymbol{\omega}_{2}\right]=\left[\begin{array}{ccc}
C_{2} & 0 & 0 \\
0 & A_{2} & 0 \\
0 & 0 & A_{2}
\end{array}\right] \cdot\left[\begin{array}{l}
\dot{\theta} \cdot \sin \varphi \\
-\dot{\varphi} \\
\dot{\theta} \cdot \cos \varphi
\end{array}\right]=} \\
& =\left[\begin{array}{l}
C_{2} \cdot \dot{\theta} \cdot \sin \varphi \\
-A_{2} \cdot \dot{\varphi} \\
A_{2} \cdot \dot{\theta} \cdot \cos \varphi
\end{array}\right], \\
& {\left[\frac{d \mathbf{G}_{2}}{d t}\right]=\left[\begin{array}{c}
C_{2} \cdot(\ddot{\theta} \cdot \sin \varphi+\dot{\theta} \cdot \dot{\varphi} \cdot \cos \varphi) \\
-A_{2} \cdot \ddot{\varphi} \\
A_{2} \cdot(\ddot{\theta} \cdot \cos \varphi-\dot{\theta} \cdot \dot{\varphi} \cdot \sin \varphi)
\end{array}\right],}
\end{aligned}
$$




$$
\left[\boldsymbol{\omega}_{2} \times \mathbf{G}_{2}\right]=\left[\begin{array}{c}
0 \\
\left(C_{2}-A_{2}\right) \cdot \dot{\theta}^{2} \cdot \sin \varphi \cdot \cos \varphi \\
\left(C_{2}-A_{2}\right) \cdot \dot{\theta} \cdot \dot{\varphi} \cdot \sin \varphi
\end{array}\right]
$$

where: $J_{2}$ is the matrix of the moment of inertia, $C_{2}$ is the equatorial moment of inertia, and $A_{2}$ is the polar moment of inertia for the projectile body.
The control moment of the nose deflection is equally the absolute value and opposite in direction for the projectile nose and projectile body, respectively. Thus:

$$
\begin{aligned}
& \frac{d \mathbf{G}_{1}}{d t}+\boldsymbol{\omega}_{1} \times \mathrm{G}_{1}+\frac{d \mathbf{G}_{2}}{d t}+\boldsymbol{\omega}_{2} \times \mathbf{G}_{2}+ \\
& =\sum \mathbf{M}_{1}+\sum \mathbf{M}_{2}=\mathbf{M}_{z 1}+\mathbf{M}_{y 1}+\mathbf{M}_{z 2}+\mathbf{M}_{y 2} .
\end{aligned}
$$

The equations of projectile dynamic and kinematic rotations about its centroid can be derived as follows.

$$
\left.\begin{array}{l}
M_{x}=C_{1} \cdot(\ddot{\alpha} \cdot \sin \beta+\dot{\alpha} \cdot \dot{\beta} \cdot \cos \beta)+C_{2} \cdot(\ddot{\theta} \cdot \sin \varphi+\dot{\theta} \cdot \dot{\varphi} \cos \varphi) \\
-M_{y}=-A_{1} \cdot \dot{\beta}+\left(C_{1}-A_{1}\right) \cdot \dot{\alpha}^{2} \cdot \sin \beta \cdot \cos \beta-A_{2} \cdot \ddot{\varphi}+\left(C_{2}-A_{2}\right) \cdot \dot{\theta}^{2} \cdot \sin \varphi \cdot \cos \varphi \\
M_{z}=A_{1} \cdot(\ddot{\alpha} \cdot \cos \beta-\dot{\alpha} \cdot \dot{\beta} \cdot \sin \beta)+\left(C_{1}-A_{1}\right) \cdot \dot{\alpha} \cdot \dot{\beta} \cdot \sin \beta+A_{2} \cdot(\ddot{\theta} \cdot \cos \varphi-\dot{\theta} \cdot \dot{\varphi} \cdot \sin \varphi)+\left(C_{2}-A_{2}\right) \cdot \dot{\theta} \cdot \dot{\varphi} \cdot \sin \varphi
\end{array}\right\},
$$

$$
\left.\begin{array}{l}
\frac{d \gamma}{d t}=\omega_{x} \\
\frac{d \varphi}{d t}=-\omega_{y} \\
\frac{d \theta}{d t}=\frac{\omega_{z}}{\cos \varphi}
\end{array}\right\} .
$$

\subsection{Angular relationship}

According to the conversion of coordinates, the above angles are not independent, so it is necessary to assess their interrelation.

$$
\mathbf{A}=\mathbf{A}_{02}^{T} \cdot \mathbf{A}_{03}=\left[\begin{array}{lll}
\mathbf{K}_{1} & \mathbf{K}_{2} & \mathbf{K}_{3}
\end{array}\right] \text { is the coordinate }
$$
transformation matrix obtained by transformation of the projectile-body coordinate to the velocity coordinate through the ground coordinate. $\mathbf{K}_{1}, \mathbf{K}_{2}$ and $\mathbf{K}_{3}$ are expressed as follows, respectively.

$$
\mathbf{K}_{1}=\left[\begin{array}{l}
{\left[\begin{array}{l}
\cos \varphi \cdot \cos \theta \cdot \cos \psi_{2} \cdot \cos \psi_{1}+\sin \theta \cdot \sin \psi_{1} \\
+\sin \varphi \cdot \cos \theta \cdot \sin \psi_{2} \cdot \cos \psi_{1}
\end{array}\right]} \\
{\left[\begin{array}{l}
\cos \varphi \cdot \sin \theta \cdot \cos \psi_{2} \cdot \cos \psi_{1}-\cos \theta \cdot \sin \psi_{1} \\
+\sin \varphi \cdot \sin \theta \cdot \sin \psi_{2} \cdot \cos \psi_{1}
\end{array}\right]} \\
{\left[\begin{array}{l}
\sin \varphi \cdot \cos \psi_{2} \cdot \cos \psi_{1}-\cos \varphi \cdot \sin \psi_{2} \cdot \cos \psi_{1} \\
{[}
\end{array}\right]}
\end{array}\right],
$$

$$
\mathbf{K}_{2}=\left[\begin{array}{l}
{\left[\begin{array}{l}
\cos \varphi \cdot \cos \theta \cdot \cos \psi_{2} \cdot \sin \psi_{1}-\sin \theta \cdot \cos \psi_{1} \\
+\sin \varphi \cdot \cos \theta \cdot \sin \psi_{2} \cdot \sin \psi_{1}
\end{array}\right]} \\
{\left[\begin{array}{l}
\cos \varphi \cdot \sin \theta \cdot \cos \psi_{2} \cdot \sin \psi_{1}+\cos \theta \cdot \cos \psi_{1} \\
+\sin \varphi \cdot \sin \theta \cdot \sin \psi_{2} \cdot \sin \psi_{1}
\end{array}\right]} \\
{\left[\begin{array}{l}
\sin \varphi \cdot \cos \psi_{2} \cdot \sin \psi_{1}-\cos \theta \cdot \sin \psi_{2} \cdot \sin \psi_{1}
\end{array}\right]}
\end{array}\right],
$$

$\mathbf{K}_{3}=\left[\begin{array}{l}\cos \varphi \cdot \cos \theta \cdot \sin \psi_{2}-\sin \varphi \cdot \cos \theta \cdot \cos \psi_{2} \\ \cos \varphi \cdot \sin \theta \cdot \sin \psi_{2}-\sin \varphi \cdot \sin \theta \cdot \cos \psi_{2} \\ \sin \varphi \cdot \sin \psi_{2}-\cos \varphi \cdot \cos \psi_{2}\end{array}\right]$.

$\mathbf{A}_{23}$ is the coordinate transformation matrix obtained by conversion of the projectile-body coordinate directly to the velocity coordinate system.
The first column of two matrices above should be equal in value because of the uniqueness of the projectile vertical axis vector. Then, equations (30) can be derived:

$$
\left.\begin{array}{l}
\sin \delta_{1}=\cos \theta \cdot \sin \psi_{1}-\sin \theta \cdot \cos \psi_{1} \cdot \cos \left(\varphi-\psi_{2}\right) \\
\sin \delta_{2}=-\cos \psi_{1} \cdot \sin \left(\varphi-\psi_{2}\right)
\end{array}\right\} .
$$

Overall, the two-rigid-body ballistic trajectory model is made up of Eqs. (15-30).

\section{Digital simulation results and discussion}

In the design process, the ballistic trajectory calculation program was realized via the standard MATLAB software package based on the two-rigid-body ballistic trajectory model, which has been established above. This program was used for calculating the external trajectory of a missile with a deflectable nose. The Four-Stage Runge-Kutta method [20-21] was also applied to numerical simulations.

4.1. Effect of shooting angle and initial velocity on external ballistic trajectory

In the simulations, the initial velocities of $170 \mathrm{~m} / \mathrm{s}$, $340 \mathrm{~m} / \mathrm{s}, 680 \mathrm{~m} / \mathrm{s}$, and $1020 \mathrm{~m} / \mathrm{s}$ were set, while the shooting angles of $2^{\circ}, 4^{\circ}, 8^{\circ}, 12^{\circ}$, and $16^{\circ}$ were used. The simulation results on firing ranges (unity: $\mathrm{m}$ ) of a standard projectile are listed in Table 1.

Table 1

Firing range for various shooting angles

\begin{tabular}{|c|c|c|c|c|}
\hline \multirow{2}{*}{ Shooting angle, ${ }^{\circ}$} & \multicolumn{4}{|c|}{ Initial velocity, m/s } \\
\cline { 2 - 5 } & 170 & 340 & 680 & 1020 \\
\hline 2 & 192.8 & 620.5 & 1527.9 & 2431.3 \\
\hline 4 & 362.9 & 1036.2 & 2122.3 & 3099.0 \\
\hline 8 & 649.4 & 1601.5 & 2809.8 & 3826.1 \\
\hline 12 & 879.8 & 1983.8 & 3236.5 & 4266.1 \\
\hline 16 & 1066.3 & 2261.7 & 3533.4 & 4568.2 \\
\hline
\end{tabular}

Fig. 3 shows the comparison of the firing ranges for various shooting angles and four values of the initial velocity. Results show that, under the set shooting conditions, the scope of firing range from 192.8 to $4568.2 \mathrm{~m}$ is obtained. With the same initial velocity, the firing range increases with the shooting angle, and when the latter is constant, the firing range increases with the initial velocity. The shooting 
angle has a significant impact on the external ballistic trajectory, and only when the shooting angle and initial velocity are optimally matched, the maximal range could be obtained.

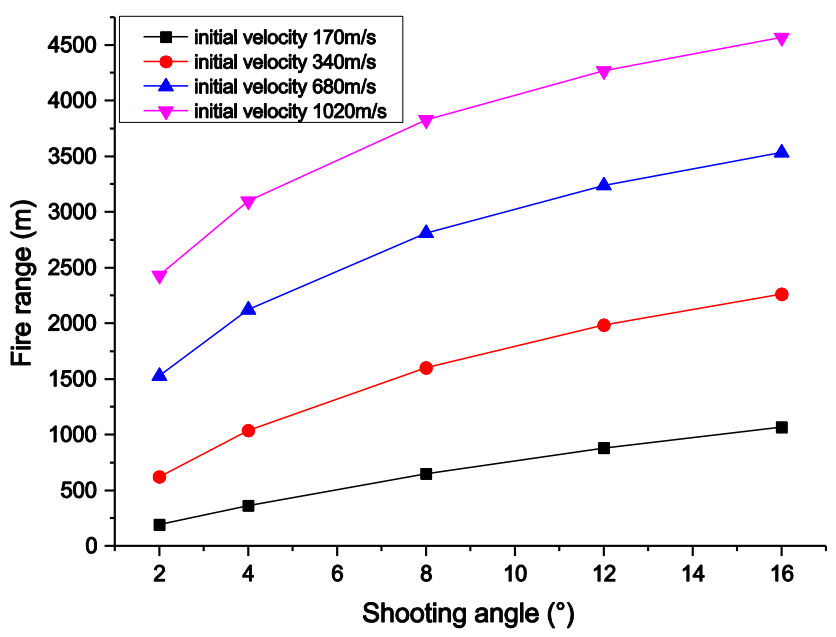

Fig. 3 Comparison of the firing ranges

4.2. Effect of nose-deflection angle on the external ballistic trajectory

In this digital simulation, we set the initial velocity as $170 \mathrm{~m} / \mathrm{s}, 340 \mathrm{~m} / \mathrm{s}, 680 \mathrm{~m} / \mathrm{s}$ and $1020 \mathrm{~m} / \mathrm{s}$ respectively; a constant shooting angle of $16^{\circ}$ was used, while the nose deflection angles in the lateral direction were $2^{\circ}, 4^{\circ}, 6^{\circ}, 8^{\circ}$ and $10^{\circ}$, respectively. The simulation results on the point of fall are summarized in Table 2.

The point of fall is expressed as three degrees of freedom $\left(X_{c}, Y_{c}, Z_{c}\right)$, whereas $Y_{c}$ values are omitted because the shooting height is $0 \mathrm{~m}$ in the point of fall.
Variation patterns of the lateral offset of points of fall are depicted in Fig.4. The results obtained strongly indicate that at the constant shooting angle, the lateral offset of the point of fall increases with the nose deflection angle for the same initial velocity. The lateral nose deflection has a little effect on the longitudinal firing range. The initial velocity at $1020 \mathrm{~m} / \mathrm{s}$ and nose deflection angle of $10^{\circ}$ can provide the maximum lateral offset of fall point, which is 108.5 m.

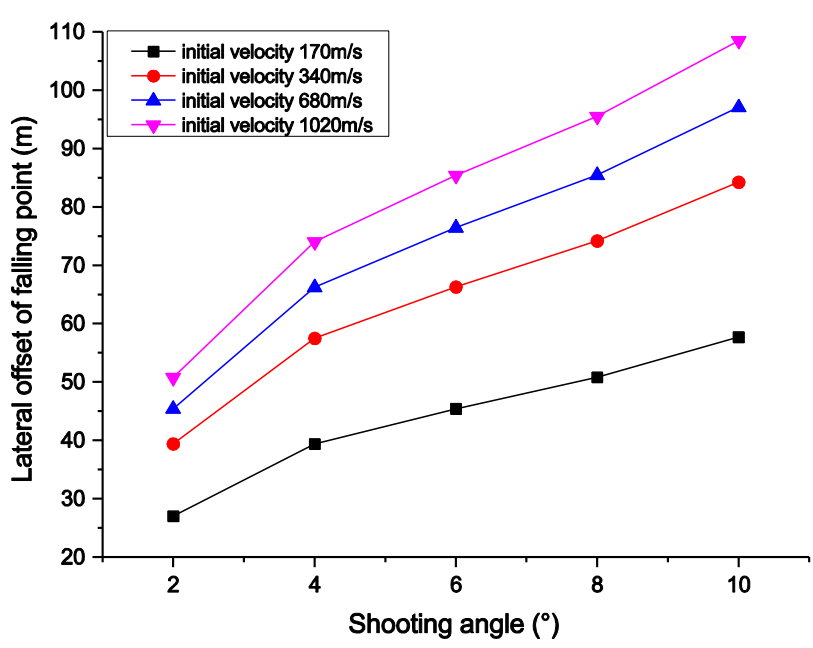

Fig. 4 Change rules of lateral offset about fall points

The windward area of projectile increases after the nose deflects at a certain angle from the projectile axis in the longitudinal direction, which results in the corresponding longitudinal firing range variation. Corrections of the firing range with different nose deflection angles in the longitudinal direction are given as show in Table 3 .

Table 2

Information on the projectile point of fall under different shooting conditions

\begin{tabular}{|c|c|c|c|c|c|c|}
\hline Initial velocity, $\mathrm{m} / \mathrm{s}$ & Nose deflection angle, $^{\circ}$ & 2 & 4 & 6 & 8 & 10 \\
\hline \multirow{2}{*}{170} & $Z_{c}, \mathrm{~m}$ & 27.0 & 39.4 & 45.4 & 50.8 & 57.7 \\
\cline { 2 - 7 } & $X_{c}, \mathrm{~m}$ & 1264.3 & 1262.8 & 1260.3 & 1255.0 & 1254.3 \\
\hline \multirow{2}{*}{340} & $Z_{c}, \mathrm{~m}$ & 39.4 & 57.5 & 66.3 & 74.2 & 84.2 \\
\cline { 2 - 7 } & $X_{c}, \mathrm{~m}$ & 1929.0 & 1921.4 & 1917.1 & 1914.6 & 1912.1 \\
\hline \multirow{2}{*}{680} & $Z_{c}, \mathrm{~m}$ & 45.4 & 66.2 & 76.4 & 85.5 & 97.0 \\
\cline { 2 - 7 } & $X_{c}, \mathrm{~m}$ & 2632.8 & 2622.0 & 2614.0 & 2611.0 & 2609.5 \\
\hline \multirow{2}{*}{1020} & $Z_{c}, \mathrm{~m}$ & 50.8 & 74.0 & 85.4 & 95.5 & 108.5 \\
\cline { 2 - 7 } & $X_{c}, \mathrm{~m}$ & 3235.2 & 3223.6 & 3212.8 & 3208.3 & 3207.8 \\
\hline
\end{tabular}

Table 3

Firing range corrections for different nose deflection angles

\begin{tabular}{|c|c|c|c|c|c|c|c|}
\hline \multirow{2}{*}{ Initial velocity, $\mathrm{m} / \mathrm{s}$} & \multirow{2}{*}{ Shooting angle, $^{\circ}$} & \multirow{2}{*}{ Firing range, $\mathrm{m}$} & \multicolumn{4}{|c|}{ Nose deflection angle, $^{\circ}$} \\
\cline { 4 - 8 } & & 2 & 2 & 4 & 6 & 8 & 10 \\
\hline \multirow{3}{*}{156} & 2 & 200 & 0.7 & 1.3 & 1.7 & 2.0 & 2.2 \\
\cline { 2 - 8 } & 5 & 400 & 2.6 & 4.7 & 6.5 & 7.7 & 8.3 \\
\cline { 2 - 8 } & 8 & 5.8 & 10.1 & 11.8 & 16.6 & 17.9 \\
\cline { 2 - 8 } & 12 & 800 & 10.2 & 17.2 & 23.1 & 28.4 & 30.8 \\
\cline { 2 - 8 } & 16 & 1000 & 16.6 & 26.5 & 35.5 & 44.5 & 48.4 \\
\hline
\end{tabular}

The digital simulation results strongly indicate that at the same firing range, the larger longitudinal nose deflection angles provide better firing range correction, and when the nose deflection angle is constant, the range correction ability is improved with the firing range increase. When the firing range is $1000 \mathrm{~m}$ and nose deflection angle is $10^{\circ}$, the maximum firing range correction of $48.4 \mathrm{~m}$ is obtained.

\section{Conclusions}

The digital simulation results obtained on the external ballistic trajectory of a projectile with the deflectable nose make it possible to draw the following conclusions. 
1. The representation of a missile with the deflectable nose by a two-rigid-body system provides an appropriate aerodynamic response of the projectile motion environment. The two-rigid-body trajectory model proposed in this study could be used for trajectory prediction of a projectile with a deflectable nose. The accurate trajectory prediction data can provide guidance to the intelligent missile design.

2. The projectile nose deflection can be used for the flight trajectory control. Research results show that the nose deflection can improve the ability of trajectory correction both in the lateral and longitudinal directions. It would be beneficial for reducing the original shooting dispersion and improving the hit probability of ammunition.

3 . In the future studies, more factors such as meteorological and geographical conditions (including latitudelongitude, earth rotation, and projectile spinning motion) should be considered in the process of ballistic trajectory model, in order to make the trajectory model more comprehensive.

4. Projectiles with a deflectable nose imply the integration of sensors with an actuator, which has high control efficiency and excellent application prospects in the ammunition system. The nose deflection actuator design based on smart materials is considered a lucrative direction for future research.

\section{Conflict of interests}

The authors declare that there is no conflict of interests regarding the publication of this paper.

\section{Acknowledgments}

The authors would like to acknowledge the financial support from the project supported by the Key Laboratory Foundation of Science and Technology on Transient Impact under Grant No.61426060101.

\section{References}

1. Yin, J. P.; Wang, Z. J. 2014. Ammunition Theory, Beijing Institute of Technology Press. 590 p. (in Chinese).

2. Yin, J. P. 2012. Multiple Explosively Formed Projectile Warhead Technology, National Defence Industry Press. 191 p. (in Chinese).

3. Goddard, R. H. 1952. Apparatus for Steering Aircraft. United States Patent Office, No. 2594766. 3 p.

4. Miller, C. G.; Gnoffo, P. A. 1982. Pressure distributions and shock shapes for a bent-nose biconic at incidence, Aero-Space Technologist, Aerothermodynamics Branch, Space Systems Divesion, AIAA Journal 20(8):1150-1152.

http://dx.doi.org/10.2514/3.7970.

5. Thomson, K. D. 1984. Wind Tunnel Tests on a TubeLaunched Missile Configuration with a Deflectable Nose Control and a Novel Wrap-Around Fin Stabiliser. Department of Defence, Defence Science and Technology Organisation, Weapons System Research Laboratory, Technical Report: WSRL-0327-TR, ADA142082.

6. Hathaway, W.; Winchenbach, G.; Krieger, J. 1999. Free-Flight Tests of 10-Degree Cones with a One Degree Articulation Angle. $37^{\text {th }}$ AIAA Aerospace Sciences Meeting \& Exhibit, Reno, Nevada, AIAA 99-0434.
7. Landers, M. G.; Hall, L. H.; Auman, L. M.; Vaughn, M. E. 2003. Deflectable nose and canard controls for a fin-stabilized projectile at supersonic and hypersonic speeds, $21^{\text {st }}$ Applied Aerodynamics Conference, Orlando, Florida, AIAA 2003-3805.

8. Shoesmith, B.; Birch, T.; Mifsud, M. Meunier, M.; Shaw, S. 2006. CFD Analysis of a Supersonic Projectile with Deflectable Nose Control. $3^{\text {rd }}$ AIAA Flow Control Conference, San Francisco, California, AIAA 20063200.

9. Khalghani, A.; Pasandideh-Fard, M.; Djavareshkian, M. H. 2016. Aerodynamic shape study of supersonic surface to surface missiles with continuous flexible nose, Journal of Mechanical Science and Technology 30(7):3183-3192. https://doi.org/10.1007/s12206-016-0512-z.

10. Zhang, B.; Wang, S. S.; Cao, M. Y.; Xu, Y. X. 2015. Simulation and analysis on aerodynamic characteristics of deflectable nose, Process in Computational Fluid Dynamics 15(5):279-289. http://dx.doi.org/10.1504/PCFD.2015.072012.

11. Li, X.; Wei, J. F.; Xu, Y. X.; Wang, S. S. 2014. Research on the influence of forehead angle on aerodynamic characteristics, Tactical Missile Technology 2014(2): 16-20. http://dx.doi.org/10.16358/j.issn.1009-300.2014.02.007.

12. Sun, X. F.; Gu, L. X.; Gong, C. L. 2012. Dynamics of a deflectable-nose missile, Technological Sciences 55(12): 3483-3494. http://dx.doi.org/10.1007/s11431-012-4984-5.

13. Sun, X. F.; Gu, L. X.; Gong, C. L. 2011. Overload control system design of a deflectable nose missile, Procedia Engineering 15(2011): 402-407. http://dx.doi.org/10.1016/j.proeng.2011.08.077.

14. Gao, Y.; Gu, L. X.; Pan, L. 2009. Modeling and simulating dynamics of missiles with deflectable nose control, Chinese Journal of Aeronauticas, 22(2009): 474479. http://dx.doi.org/10.1016/S1000-9361(08)60128-4.

15. Xu, M. Y. 2003. Advanced Exterior Ballistics, Advanced Education Press. 181 p. (in Chinese).

16. Wang, Z. Y. 2004. Theory and Method of External Ballistics Design, Science Press. 133 p. (in Chinese).

17. Han, Z. P. 2014. Exterior Ballistics of Projectile and Rockets, Beijing Institute of Technology Press. 673 p. (in Chinese).

18. Li, J. L.; Wu, J. N.; Yan, S. Z. 2013. Conceptual Design of Deployment Structure of Morphing Nose Cone. Mathematical Problems in Engineering, vol. 2013, Article ID590957. http://dx.doi.org/10.1155/2013/590975.

19. Hawley, P. A.; Blauwkamp, R. A. 2010. Six-Degreeof-Freedom digital simulations for missile guidance, navigation, and control, Johns Hopkins Apl. Technical Digest 29(1): 71-84.

20. Wang, M. Z. 2009. Artillery Applied Exterior Ballistics and Simulation, National Defence Industry Press. 343 p. (in Chinese).

21. Wei, F. H.; Wang, Z. J.; Wu, G. D. 2006. A new method of ballistic correction by controlling the nose angle of rocket, Journal of Projectiles, Rockets, Missiles and Guidance 26(2): 928-930 (in Chinese). http://dx.doi.org/10.15892/j.cnki.djzdxb.2006.s9.021. 
Yongjie XU, Zhijun WANG, Fangdong DONG

\section{BALLISTIC TRAJECTORY MODELING FOR MISSILE WITH DEFLECTABLE NOSE}

\section{S u m mary}

With the development of science and technology, significant changes have taken place in the mode of modern wars. Application of military operating concepts such as 'surgical precision strike' and 'decapitation strike' make higher demands on precision-strike weapons. Ballistic trajectory correction ammunition is being rapidly developed due to its lucrative combination of low cost, high cost-effectiveness ratio, high damage rate, and applicability of existing inventory ammunition. Ballistic control technology has distinct advantages both in cost saving and improvement of the ammunition operational performance. Nose deflection is a feasible, effective, and fast-response flight control mode.
The nose part of a projectile can be deflected at a certain angle relative to the projectile body axis, including a pressure difference between the windward and leeward sides of the warhead and generating the respective aerodynamic control force. In this study, a two-rigid-body trajectory model is established based on the multiple rigid body system theory. The proposed model is used to predict the flight trajectory of a projectile with the deflectable nose. Finally, the nose deflection effect on the ballistic trajectory variation is analyzed. The research results obtained provide the theoretical basis for the development of adaptive control smart ammunition and its engineering applications.

Keywords: mechanics of rigid bodies, nose deflection, trajectory model, digital simulation.

Received May 10, 2019

Accepted October 14, 2020

This article is an Open Access article distributed under the terms and conditions of the Creative Commons Attribution 4.0 (CC BY 4.0) License (http://creativecommons.org/licenses/by/4.0/). 